\title{
Theory and Practice of Optimum Correction using Restored Track Irregularity Waveform
}

\author{
Masako KAMIYAMA \\ Researcher, Track Dynamics Lab., Fundamental Research Div.
}

\author{
Atsushi FURUKAWA \\ Engineer, Track Geometry and Maintenance G., \\ Track Technology Development Div., Technological Development Dept.
}

\author{
Akiyoshi YOSHIMURA \\ Professor, Tokyo Engineering University
}

\begin{abstract}
We have developed a new theory for correcting track irregularities by applying their restored waveforms and carried out experimental levelling and lining work. In this theory, the optimum shifting / lifting values are obtained as solutions for a typical quadratic programming problem. Restrictions on shifting rails are formulated as constraint conditions of the optimization problem. Furthermore, in the case of levelling work, a designed cross level can be realized. From experimental work on ballasted and slab track, the effectiveness of this theory has been confirmed. In the range of long wavelengths up to $50 \mathrm{~m}$, the amplitude of track irregularity has decreased.
\end{abstract}

Keywords : track maintenance, track irregularity, tamping machine, restored waveform, optimization

\section{Introduction}

Train speeds have continuously been increased in Japan since the privatization of Japanese National railways, which requires increasingly longer wavelengths of track irregularity that must be corrected.

However, it is difficult to correct track irregularities, when their wavelength is longer than $30 \mathrm{~m}$. By the "relative reference frame method" which is commonly used to correct ballasted track irregularities, about $40 \%$ of irregularities remain after tamping work when the wavelength is $30 \mathrm{~m}$, and more than $50 \%$ remain when the wavelength is $50 \mathrm{~m}$. In the case of the "absolute reference frame method" on the other hand, the optimum track geometry after correction work can be calculated from the actual track geometry surveyed before the work, and allows for almost perfect correcting of track irregularities. However, this method requires surveying the actual track geometry beforehand, which involves very laborious and time-consuming work. For these reasons, a more effective and easier method to correct track irregularities has been needed.

A well-known idea to solve this problem is to apply the "restored waveform" of track irregularity instead of surveying the actual track irregularity (e.g. Yoshimura $\left.{ }^{1)}\right)$. With this idea, track irregularities over a wide range of wavelengths can be corrected better than by the relative reference frame method. A restored waveform is calculated from track irregularity data measured with a track inspection car, as an approximate waveform of actual track irregularity. A characteristic of this calculation depends on the measuring system mounted on the track inspection car or heavy tamping machines. The restored waveform can be regarded approximately as the actual track irregularity whose wavelength ranges from several meters to nearly $100 \mathrm{~m}$.

However, calculating shifting/lifting values from the restored waveform is difficult, because there are a number of restrictions on shifting rails on the actual track.

The authors have developed a new calculation theory for rail shifting values in order to cope with the restrictions, and to adjust it for applications with heavy tamping machines. The former applies the well-known optimization problem, and the latter formulates a series of processes, which convert rail shifting values to controlling data for a tamping machine.

In this paper, we describe an outline of the theory to calculate the optimum shifting values, process it to adjust for heavy tamping machines and produce results from experimental work.

2. Theory of calculating the optimum shifting values and its adjustment to tamping machines

\section{1 Features of theory}

Requirements for the optimum shifting values for correcting track irregularities are as follows.

a) When we correct track irregularities in the longitudinal level, we must realize the cross level design at the same time. However, measured track data in the longitudinal level of both rails does not include all information on irregularities in the actual cross level. Hence, we can not obtain values of irregularity in the cross level from measured irregularities in the longitudinal level. This is because inspection cars measure both rails in the longitudinal level separately. 
b) Many restrictions on the shifting operation exist on actual tracks. For example, only a lifting-up operation is allowed in the levelling work on ballasted tracks. Furthermore, there are other restrictions such as those related to track clearance, level crossings, tunnels, platforms of stations and boundaries of ballast-less bridges. This theory satisfies these requirements as follows.

a) Restored waveforms of both rails in the longitudinal level are converted into new waveforms based on a common coordinate by applying measured actual track data in the cross level.

b) The problem of calculating shifting values is formulated as a typical quadratic programming problem in the optimization theory. In addition, restrictions mentioned above are considered as constraint conditions in this formulation.

\section{2 Restoration of actual track irregularity}

In this theory, we use data measured with a 3-point measurement system instead of surveyed data, and these data include a component due to the fundamental track geometry in addition to that due to the true track irregularity. With a view to correcting only the true track irregularity without affecting the fundamental track geometry, fundamental data must be extracted from the measured data before the calculation of the shifting values. The remainder of the measured data after the extraction is a true target component.

However, this remainder is different from the actual track irregularity waveform. This is because the magnitude gain of the 3-point measurement system is different for different wavelength components. In the case of the $10 \mathrm{~m}$-chord versine measurement system, for example, the amplitude gain is zero at each wavelength of $10 / 2 k(k=$ $1,2 \ldots)$ of track irregularities. Figure 1 shows a frequency response function of the $10 \mathrm{~m}$-chord versine measurement system. In other words, we can not calculate the true track geometry from this data, because the measured track data with the $10 \mathrm{~m}$-chord versine does not have all information on the track geometry.

To calculate an approximate track irregularity waveform, therefore, the measured track data of a partial range of wavelength is restored through an extra filter whose frequency characteristic corresponds to the inverse of that of the measurement system (e.g. Yoshimura ${ }^{2)}$ ). To correct the track irregularity, the wavelength range of restoration will usually be selected from that of a usable amplitude. In the case of $10 \mathrm{~m}$-chord versine, the speci-

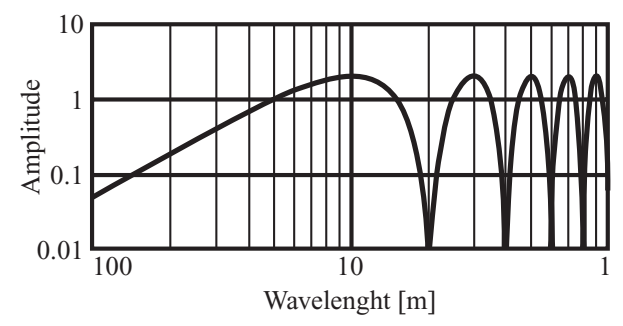

Fig. 1 Frequency response function of $10 \mathrm{~m}$-chord versine measurement fied range of restoration wavelength may be selected from $6 \mathrm{~m}$ to $100 \mathrm{~m}$ because it has a large influence on vehicle vibration.

Then, the restored component of track irregularity will be corrected and other components will be left as the remainder of restoration. The relation among the actual track irregularity $x_{i}(i=$ position $)$, restored portion $x_{R, i}$ and remainder $x_{N, i}$ are expressed as follows.

$$
x_{i}=x_{N, i}+x_{R, i}
$$

Most of the values of $x_{N, i}$ and $y_{N, i}$ consist of waveforms whose wavelength is longer than $100 \mathrm{~m}$. They are not the targets of our correcting work.

\section{3 Common base line for correcting track irregularities in the longitudinal level}

During levelling work, the designed cross level must be realized, while the gauge is unchanged in the lining work on ballasted tracks, because the rails are fastened to sleepers. As inspection cars measure track irregularities of the left and right rails separately, the base lines of coordinates of the heights of these rails are different.

For this problem, by using the measured data of the cross level, a new common base line $s_{N, i}$ is defined as the average of the remainders of restoration of the two rails as shown in Fig. 2 (e.g. Yoshimura ${ }^{3)}$ ).

$$
s_{N, i}=\frac{1}{2}\left(x_{N, i}+y_{N, i}\right)
$$

where $s_{N, i}$ : Common base at $i(0 \leq i \leq N-1)$

$x_{N, i}$ : Remainder of the left rail at $i$

$y_{N, i}$ : Remainder of the right rail at $i$

We define this $s_{N, i}$ as the common base line for correcting the longitudinal level irregularity.

Then, new longitudinal level irregularities are defined on the new base line as eq. (3).

$$
\begin{aligned}
& z_{x, i}=x_{R, i}-s_{N, i} \\
& z_{y, i}=y_{y, i}-s_{N, i}
\end{aligned}
$$

The values of $z_{x, i}$ and $z_{y, i}$ are calculated with measured track data in the cross level, the designed cross level and restored track irregularities in the longitudinal level of both rails.

Next, in the case of levelling work on ballasted tracks, only lifting-up operation is allowed, in contrast to the lining work in which rails can be shifted in either direction, right or left. Hence a lifting value at each position can not be a negative value. Then, an initial longitudinal level irregularity is defined as eq. (4), $z_{i}$.

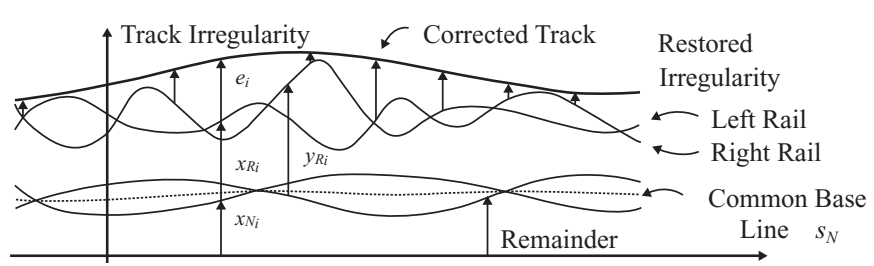

Fig. 2 New base line for levelling work 
$z_{i}=\max \left\{z_{x, i}, z_{y, i}\right\}$

The optimum lifting values of the higher rail is calculated as the difference between an ideal track geometry and $z_{i}$.

\section{4 Optimization of rail shifting value}

\section{4. 1 Evaluating the smoothness of track geometry}

To obtain the optimum track shifting/lifting values, an evaluation value of track geometry is necessary. Moreover, the track geometry of each wavelength must be evaluated individually, because the influence on riding comfort depends on the wavelength of track irregularity.

To evaluate the smoothness of track irregularity waveforms, the power spectrum density function is often used. However, calculation of the optimum track irregularity waveform in the frequency domain is impractical when all constraint conditions are to be satisfied.

On the other hand, this calculation is equivalent to that in the space domain. It is performed with a digital filter, which has characteristics equivalent to those of an evaluation in the frequency domain.

Then, the evaluation value of the smoothness of track geometry ' $U$ ' is obtained as the mean square of the track irregularity waveform which is calculated with a digital filter. It is defined as:

$$
U=\frac{1}{N} \sum_{n=0}^{N+p-2}\left\{\sum_{i=0}^{p-1} h_{i}\left(z_{n-i}+e_{n-i}\right)\right\}^{2}
$$

where $e_{i}$ : Shifting quantity at $i(0 \leq i \leq N-1)$

$z_{i}$ : Restored irregularity at $i$

$h_{i}:$ Impulse response of digital filter with length $p$

This coefficient $U$ is named the smoothness evaluation coefficient, and the digital filter $h_{n}$ is named the smoothness evaluation filter. When the value of $U$ is small, the track irregularity waveform is smoother.

\section{4. 2 Evaluation of the total amount of correcting work}

To evaluate the total amount of correcting work, the mean square value of shifting values $E$ shall be calculated. This coefficient is desired to be smaller. This is because a large shifting value may induce a larger error in rail shifting with a tamping machine.

The value of $E$ is defined as:

$$
E=\frac{1}{N} \sum_{i=0}^{N-1} e_{i}^{2}
$$

2. 4. 3 Correcting work performance evaluating function

In general, smoothness of track irregularity after levelling/lining work and small shifting values are incompatible with each other. This is because smoother track geometry requires large amounts of shifting work.

In order to specify the importance of the two factors, we shall define a function $T$ that evaluates the overall performance of correcting work. This is defined as:

$$
T=\alpha U+\beta E
$$

Where $\alpha$ and $\beta$ are called weighting coefficients, which enable us to specify the importance of these factors.

\section{4. 4 Constraints condition}

There are many restrictions in the shifting operation on tracks as mentioned above, and these can be considered as constraint conditions in the optimization problem.

$b_{\text {low }, i} \leq e_{i} \leq b_{u p, i}$

where $b_{\text {low, } i}$ : Lower limit of constraint at $i$ $b_{u p, i}:$ Upper limit of constraint at $i$

\section{4. 5 Formulation of optimization problem}

The minimization problem of this object function under the constraints mentioned above can be considered as a typical quadratic programming problem in the optimization theory (e.g. Yoshimura ${ }^{3)}$ ). This is defined as:

minimize $T(\boldsymbol{e}) \quad$ subject to $b_{\text {low }, i} \leq e_{i} \leq b_{u p, i}$ $\cdots(9)$

This optimization problem can be solved based on the well-known active set method of constraints.

\section{5 Adjustment to tamping machines}

When correcting track irregularities on ballasted tracks, a heavy tamping machine is used. Figure 3 shows a principle of correction with a machine with the trailing point on the corrected track, and the leading point on the old track.

In order to shift rails to the shifting values calculated before the correcting work without affecting the fundamental track geometry or the remainder of restoration, an adjustment quantity, $\Delta m$, must be left as shown in Figure 3. This is calculated as follows.

$$
\Delta m_{i}=T\left[x_{B, i}\right]+T\left[x_{N, i}\right]+M_{2}\left[x_{R, i}+e_{i}\right]
$$

Where $x_{B, i}$ is the fundamental track geometry measured by the 3-point measurement system. Both $M_{1}$ and $M_{2}$ are linear operators which represent the equation describing the 3-point measurement method.

$M_{1}[\bullet]$ is for the measurement system.

$M_{2}[\bullet]$ is for the correction system.

$T[\bullet]$ is also a linear operator representing the transformation from $M_{1}[\bullet]$ to $M_{2}[\bullet]$ defined as $M_{2}[\bullet]=T\left[M_{1}[\bullet]\right]$.

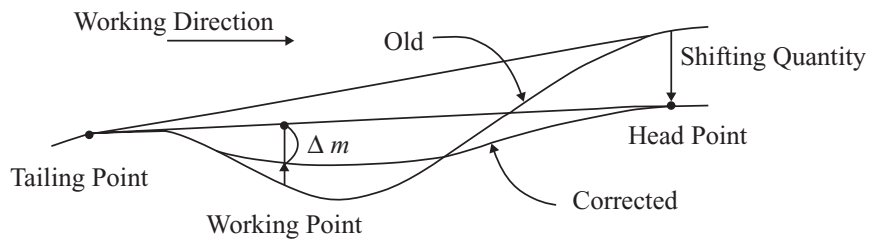

Fig. 3 Operating method of a tamping machine in the new method 
When we correct track irregularities by this correcting theory, we control a heavy tamping machine with two values. One is the calculated shifting value $e_{i}$, and the other is $\Delta m$. These must be converted into the values that fit the operating system of the machine in use.

\section{Verification with experimental work}

To confirm the theory mentioned in Chap. 2, we carried out experimental work in JR Hokkaido. In this chapter, we will describe the results and estimates made from this work.

\section{1 Lifting work at Hakodate Main Line}

\section{1. 1 Outline of the lifting work}

Table 1 shows an outline of the experimental lifting work.

\section{1. 2 Position-matching between measured track irregularity and the rail in the field}

In calculating the optimum shifting values using track irregularity data measured with an inspection car, it is very important to have position-matching between the inspected track irregularity and the rail in the field. This is because, if some difference between the two positions exists, a shifting value for one position is given to an incorrect position. As a result, the ideal track geometry will not be obtained. Especially in the case of lining work in a curve section, the fundamental track geometry will be affected.

In this experimental work, we measured the track irregularity with simple inspection equipment just before the work. Then, we calculated the cross-correlation coefficient of the two track irregularities, which were measured with an inspection car and simple equipment. Fig-

Table 1 Outline and condition of the lifting work

\begin{tabular}{l|l}
\hline Alignment & Includes two right curve sections. \\
\hline Gradient & $-3 \%$ constant \\
\hline Tamping machine & $\begin{array}{l}\text { Plasser \& Theurer 07-16 } \\
\text { Control values are entered manually } \\
\text { into the machine. }\end{array}$ \\
\hline $\begin{array}{l}\text { Range of restoration } \\
\text { wavelength }\end{array}$ & $6 \mathrm{~m} \sim 60 \mathrm{~m}$ \\
\hline$\beta / \alpha$ & $10^{-8}$ \\
\hline
\end{tabular}

ure 4 shows the cross-correlation coefficient. From this figure, we can estimate that the difference between the two positions of the irregularities is $3 \mathrm{~m}$. Hence, the position of the inspected track irregularity waveform had shifted $3 \mathrm{~m}$ before the calculation of optimum lifting values.

\section{1. 3 Result and estimation of the work}

Figure 5 shows the optimum solution of lifting values. Figure 6 shows track irregularities in the longitudinal level inspected before the lifting work (thin lines), and immediately after the work (bold lines). Comparison of these waveforms indicates the effectiveness of the new theory, since the amplitude of irregularity waveform clearly decreases. Figure 7 shows power spectrum densities of track irregularities before and after the work. And Fig. 8 shows the decreasing ratio of amplitude at each frequency before and after the work. In the range of long wavelengths up to $50 \mathrm{~m}$, the power spectrum density becomes smaller than the theoretical ratio obtained by the relative reference frame method.

\section{1. 4 Errors in the lifting work}

If the work is carried out perfectly, the track irregularity would be approximately zero as shown in Fig. 5 . However, track irregularities are actually left as shown by the bold lines in Fig. 6 . This is caused by practical

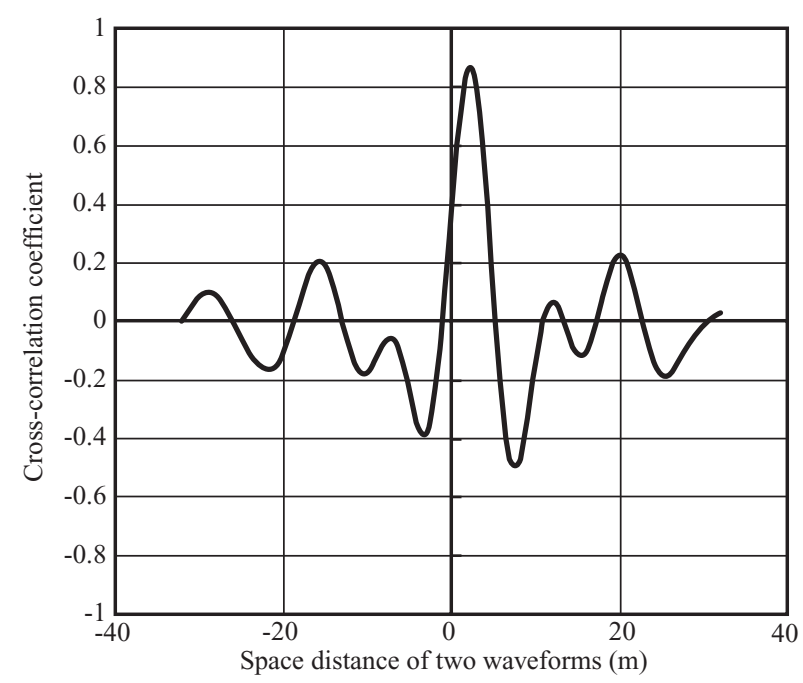

Fig. 4 Cross-correlation coefficient of two waveforms

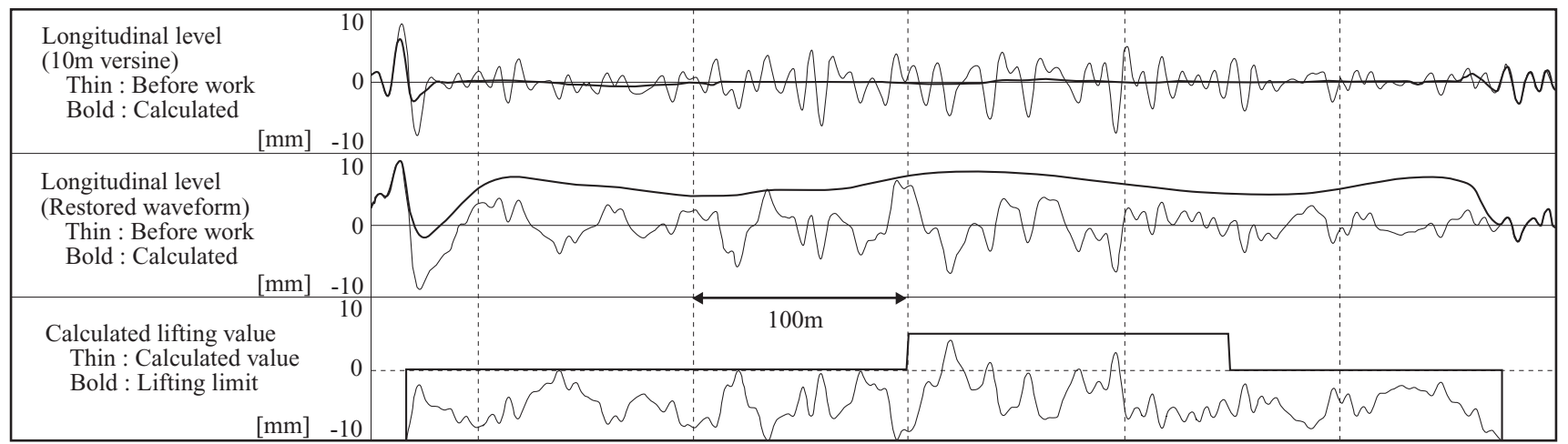

Fig. 5 The optimum solution for lifting work 


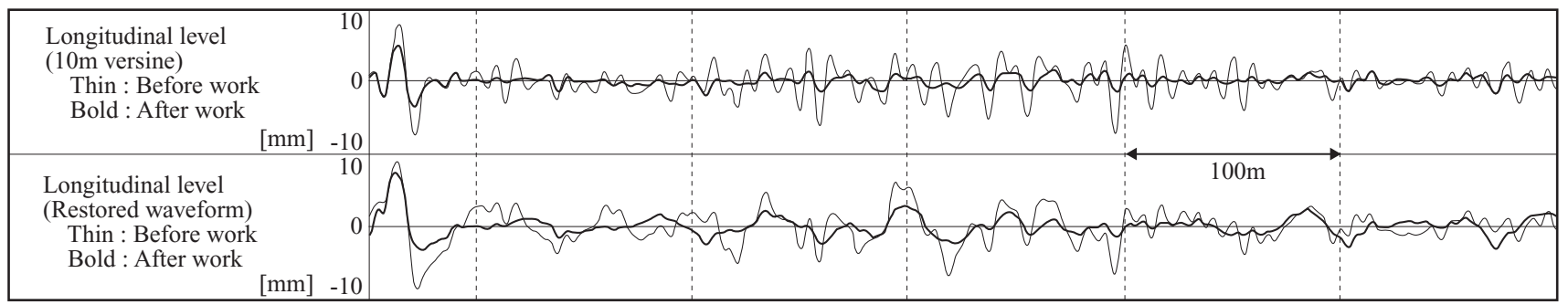

Fig. 6 Waveforms of track irregularities before and after the lifting work

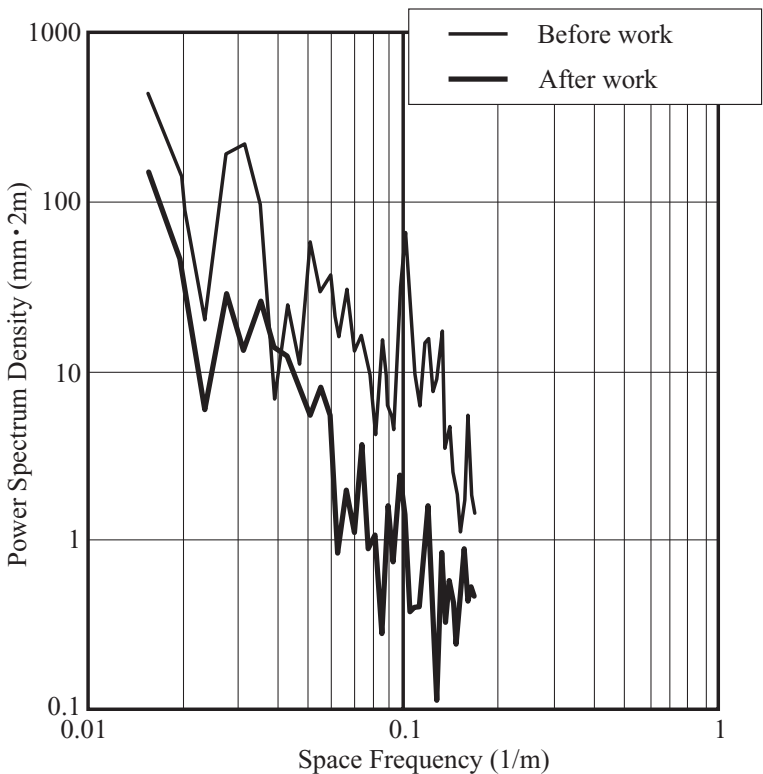

Fig. 7 Power spectrum densities

errors, which include immediate settlement of rails after the lifting work. These errors are considered when determining new lifting values to correct the remaining track irregularity as shown in Fig. 9. (They are not actual errors, but only approximate values.) In addition, a practical lifting value will be presumed as a subtraction between a calculated lifting value at the beginning and a presumed practical error.

The calculated lifting values and the practical lifting values are plotted in Fig. 10. The average ratio of practical to calculated lifting values is approximately 0.7 . With this result, we can present the following two hypotheses.

(1) Lifting values should not be too large, because practical errors will become large.

(2) If $30 \%$ is added to the calculated lifting values, the remaining track irregularity waveform may be smoother than this result.

But this value of 0.7 will change when conditions of ballast stones are changed. Hence we shall confirm these two hypotheses by performing experimental work in the future.

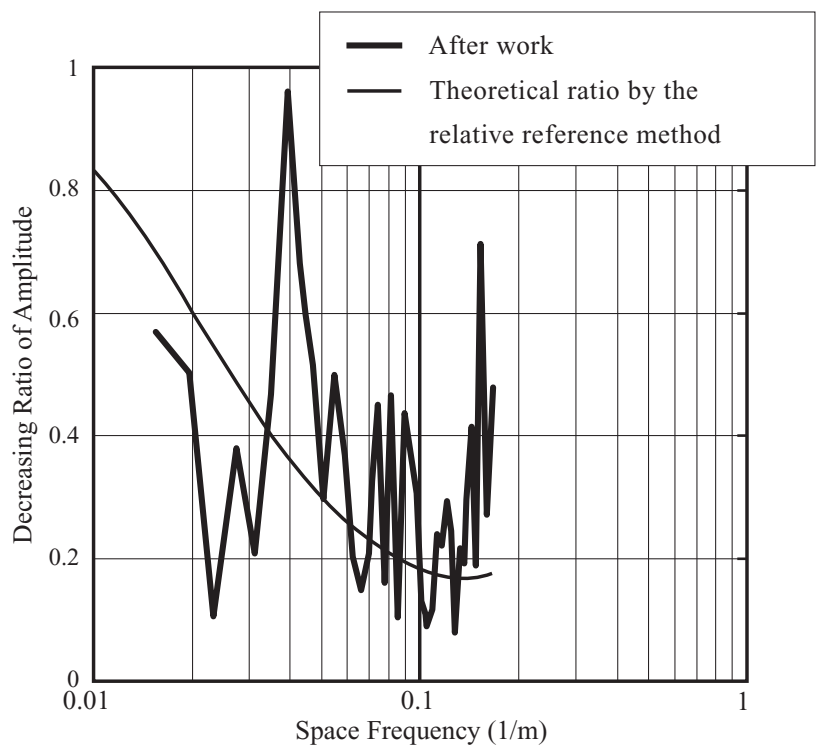

Fig. 8 Decreasing amplitude ratio

\section{2 Lining work at the Muroran Main Line}

In the case of slab tracks, the growth rate of track irregularity is slower than that of ballasted tracks. However, once track irregularity occurs, it is difficult to calculate the ideal track geometry even if the actual track irregularity waveform is known, because the movable range of rails is different at different fastenings.

In such a case, the above new theory is effective, because each movable range is formulated as a constraint condition in this theory.

We have practiced experimental lining work to confirm the effectiveness of this theory on slab track. Figure 11 shows track irregularities before the work, calculated shifting value, movable range at each position, and track irregularities after the work.

The amplitude of track irregularity was decreased by the work which proves that this theory is effective on slab tracks.

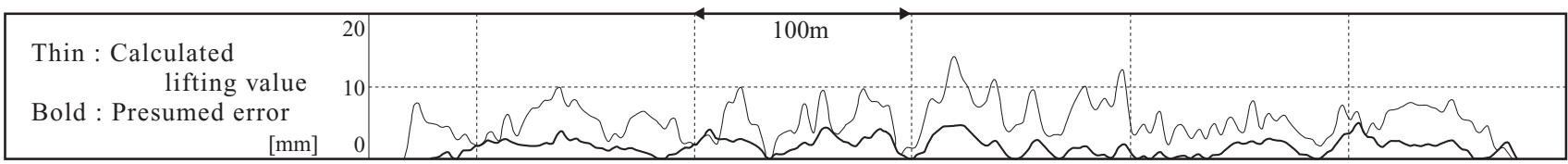

Fig. 9 Presumed practical errors 


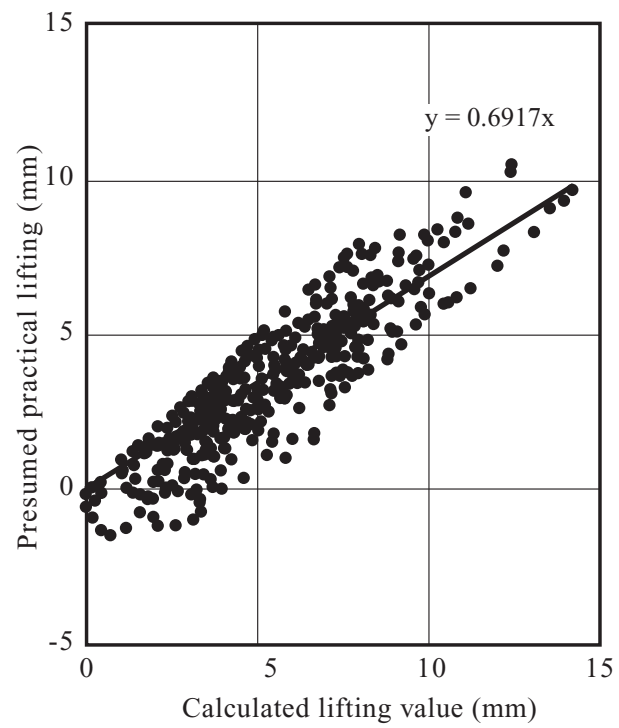

Fig. 10 Calculated lifting values and practical lifting values

\section{Conclusion}

We have developed a new theory for correcting track irregularities by applying their restored waveform and carried out experimental lifting and lining work.

In this theory, the optimum shifting values are obtained as solutions for a typical quadratic programming problem. In the case of levelling work, the optimum longitudinal geometry is obtained at the same time as the designed cross level.

Restrictions on shifting rail are formulated as constraint conditions of the optimization problem.

From experimental work on ballasted and slab track, the effectiveness of this theory has been confirmed. In the range of long wavelengths up to $50 \mathrm{~m}$, the amplitude of track irregularity is decreased.

Errors in the lifting work with a tamping machine are approximately $30 \%$ of the calculated lifting values. Therefore, if the calculated lifting values are increased by $30 \%$, remaining track irregularity may be decreased.

In the next experimental work, accuracy with smaller errors and increased labor-saving work will be the focus, especially in regard to position-matching and the method of inputting control values into the tamping machine.

\section{Acknowledgement}

The authors express their sincerest thanks to Track

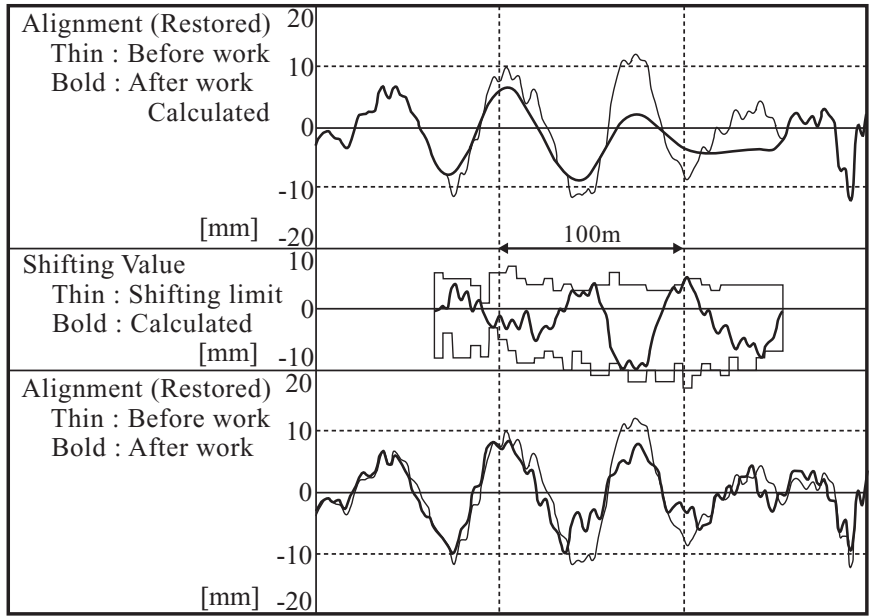

Fig. 11 Results of lining work on slab track

Maintenance Div., Iwamizawa Track Maintenance Office and Muroran Track Maintenance Office, JR Hokkaido, who have kindly co-operated during our experimental work.

\section{References}

1) Yoshimura, A. : "A new method for repairing railway track irregularities using levelling and lining machines", Proc. of COMPRAIL 96, Berlin, Germany, Vol. 1, pp. 51-60, 1996.

2) Yoshimura, A. : "Theory and practice for restoring an original waveform of a railway track irregularity", Quarterly Reports of RTRI, Vol.36, no.2, pp. 95-94, 1995.

3) Yoshimura, A. et al. : "Mathematical Analysis and Optimization for Repairing Railway Track Irregularities Using Restored Waveforms" (in Japanese), Transactions of the Japan Society for Industrial and Applied Mathematics, Vol.8, No.1, pp.107-133, 1998

4) Yoshimura, A. et al : "Database system for a railway track maintenance management, Micro LABOCS- II + and its Application", Proc. of COMPRAIL 92, Washington DC, USA, Vol. 1, pp. 149-160, 1992.

5) Kamiyama, M. et al : "Software for Railway Track Irregularity Correction Using Heavy Tamping Machine", Proc. of COMPRAIL 98, Lisbon, Portugal, Vol. 1, pp.23-32, 1998. 